\section{RMD Open}

Rheumatic \&

Musculoskeletal Diseases

\title{
Risk of venous thromboembolism in
} immune-mediated inflammatory diseases: a UK matched cohort study

James Galloway, ${ }^{1}$ Kevin Barrett, ${ }^{2}$ Peter Irving, ${ }^{3,4}$ Kaivan Khavandi, ${ }^{5}$ Monica Nijher, ${ }^{5}$ Ruth Nicholson, ${ }^{5}$ Simon de Lusignan, 6,7 Maya H Buch (1) 8,9

To cite: Galloway J, Barrett K Irving $\mathrm{P}$, et al. Risk of venous thromboembolism in immune-mediated inflammatory diseases: a UK matched cohort study. RMD Open 2020;6: e001392. doi:10.1136/ rmdopen-2020-001392

- Supplemental material is published online only. To view please visit the journal online (http://dx.doi.org/10.1136/rmdo pen-2020-001392).

Received 8 July 2020 Revised 28 August 2020 Accepted 5 September 2020

Check for updates

\section{(c) Author(s) (or their} employer(s)) 2020. Re-use permitted under CC BY-NC. No commercial re-use. See rights and permissions. Published by BMJ.

For numbered affiliations see end of article.

Correspondence to

Maya H Buch; maya.buch@ manchester.ac.uk

\section{ABSTRACT}

Objectives To describe the risk of venous

thromboembolism (VTE), and risk factors for VTE, in people with immune-mediated inflammatory diseases (IMID)

(ulcerative colitis, Crohn's disease (CD), rheumatoid arthritis (RA) and psoriatic arthritis (PsA)), compared with a matched control population.

Methods A total of 53378 people with an IMID were identified over 1999-2019 in the UK Royal College of General Practitioners (RCGP) Research and Surveillance Centre (RSC) primary care database and were matched to 213512 people without an IMID. The association between the presence of any IMID, and each IMID separately, and risk of VTE was estimated using unadjusted and multivariableadjusted Cox proportional hazards models. The prevalence of VTE risk factors, and associations between VTE risk factors and risk of VTE, were estimated in people with and without an IMID.

Results People with an IMID were at increased risk of VTE (adjusted HR [aHR] 1.46, 95\% Cl 1.36,1.56), compared with matched controls. When assessing individual diseases, risk was increased for CD (aHR 1.74, 95\% Cl 1.45 to 2.08), ulcerative colitis (aHR $1.27,95 \% \mathrm{Cl} 1.10$ to 1.45 ) and RA (aHR $1.54,95 \% \mathrm{Cl} 1.40$ to 1.70) but there was no evidence of an association for PsA (aHR 1.21, 95\% Cl 0.96 to 1.52). In people with an IMID, independent risk factors for VTE included male sex, overweight/obese body mass index, current smoking, history of fracture, and, across study follow-up, abnormal platelet count.

Conclusions VTE risk is increased in people with IMIDs. Routinely available clinical information may be helpful to identify individuals with an IMID at increased future risk of VTE.

Observational study registration number Clinicaltrials. gov (NCT03835780).

\section{INTRODUCTION}

Venous thromboembolism (VTE), comprising pulmonary embolism (PE) and deep vein thrombosis (DVT), is relatively common, with an incidence in the general population of around 3 cases per 1000 patient years. ${ }^{1}$ It is associated with significant morbidity and mortality. $^{23}$

Inflammation increases the risk of VTE, ${ }^{4}$ and observational data demonstrate higher

\section{Key messages}

What is already known about this subject?

- Risk of venous thromboembolism (VTE) is increased in people with immune-mediated inflammatory diseases (IMIDs; ulcerative colitis, Crohn's disease, rheumatoid arthritis and psoriatic arthritis) compared with the general population, but differences in VTE risk have not been systematically compared across these conditions.

- The magnitude and relevance of VTE risk from traditional VTE risk factors (such as obesity, fractures, and use of specific medications) in IMIDs is unknown.

What does this study add?

- In over 266890 people, risk of VTE was increased to a similar degree in people with ulcerative colitis, Crohn's disease and rheumatoid arthritis. For psoriatic arthritis, risk was not significantly increased, likely due to lack of statistical power.

- Risk factors identified in people with IMIDs include male sex, overweight/obese BMI, smoking, fractures, use of corticosteroids and oral contraceptives, and abnormal platelet count.

How might this impact on clinical practice?

- Knowledge of specific risk factors in people with immune-mediated inflammatory diseases can help identify those susceptible to developing VTE.

VTE rates in individuals with immunemediated inflammatory diseases (IMID) including ulcerative colitis (UC), Crohn's disease (CD) and rheumatoid arthritis (RA) compared with the general population. ${ }^{5-9}$ Evidence for VTE risk in other inflammatory diseases, including psoriatic arthritis (PsA) is more limited. ${ }^{6}$ Risk factors for VTE have been well described in the general population, and include obesity, fractures, surgery, use of oral corticosteroids and hormone therapies. $^{1011}$ and high platelet count which has been reported to be a risk factor for VTE in hospital inpatients, ${ }^{12}$ and is recognised as 
a marker of inflammation in inflammatory bowel disease and RA. ${ }^{13} 14$ There has however been little systematic interrogation of whether VTE risk factors convey the same risk in individuals with and without an IMID. ${ }^{15}$

In this study, we set out to use a large UK primary care database to establish the excess risk of VTE in people with an IMID (UC, CD, RA and PsA) compared with a control population without any of these conditions. We then compared the prevalence of traditional VTE risk factors in people with and without an IMID, and the associations between these features and future risk of VTE.

\section{METHODS}

\section{Study design}

We performed a cohort study using matched populations to compare VTE risk in adults with an IMID (UC, CD, RA and PsA) and controls between 1999 and 2018 inclusive, using UK population-based primary care data.

\section{Data source}

Data were sourced from the Royal College of General Practitioners Research (RCGP) and Surveillance Centre (RSC) database. RCGP RCS derives data from a representative network of general practices distributed across England, currently covering a registered population of 2 million people. ${ }^{16}$ RCGP RSC contains information on demographics, clinical features and diagnoses, laboratory tests and prescriptions, and studies using RCGP RSC data have been published across a range of chronic diseases. ${ }^{17-20}$

\section{Study population}

Adults (aged $\geq 18$ ) were eligible for inclusion if registered with a general practice between January 1, 1999 and December 31, 2018, with at least one consultation over that period (to minimise the impact of 'ghost' patients), and no history of VTE.

\section{Definition of the exposed cohort with IMID}

The exposed cohort was defined as all individuals with an existing or incident diagnosis of UC, CD, RA or PsA in the RCGP RSC database over the study period. UC, CD and RA were identified using Read diagnostic codes and algorithms previously validated by review of individual patient records or collection of questionnaires from general practitioners in UK primary care studies. ${ }^{21-25}$ In the absence of a validated method to identify the presence of PsA from UK primary care data, this was identified using a Read code list generated in accordance with published guidance. ${ }^{26} 27$ The index date for start of follow-up for exposed individuals began on the latest of the date of diagnosis indicated by first diagnostic code, January 1,1999 , or 180 days after practice registration.

\section{Definition of the matched unexposed cohort}

People with an IMID were matched at their index date with four unexposed individuals at general practice level by current age (per year), sex and years since practice registration (nearest neighbour matching, with replacement). The eligible pool of unexposed individuals at each index date comprised individuals registered at that date with no history of an IMID and at least 1 year of follow-up in RCGP RCS (to minimise the risk they had a non-recorded existing IMID diagnosis). Follow-up for each matched individual started on the index date of their matched case. Individuals with an incident diagnosis of an IMID during the study period were included in the pool of eligible unexposed individuals, but if matched were censored on the date of their diagnosis of an IMID; that is, these individuals were eligible to contribute to unexposed person time before their diagnosis of an IMID. Follow-up for each individual ended at the earliest of the study end-date (December 31, 2018), the date an individual was transferred from an included practice, date of death or the date an individual developed an outcome of interest.

\section{Outcome measures}

The primary outcome was a diagnosis of VTE (a composite of PE or DVT). The secondary outcomes were individual diagnoses of PE and DVT. When both PE and DVT occurred on the same date this was classified as PE. Outcomes were identified using updated Read code lists previously validated by review of patient records and provision of general practitioner questionnaires. ${ }^{28}$ Risk of each outcome was compared between individuals with an IMID and the matched control population, and between individuals with UC, CD, RA and PsA and their matched counterparts.

\section{Recorded characteristics and VTE risk factors}

Baseline features comprised sociodemographic characteristics, clinical VTE risk factors, comorbidities and medication use. VTE risk factors were selected based on existing literature demonstrating an established association with $\mathrm{VTE}^{610}$ and clinical expertise. Clinical VTE risk factors were body mass index (BMI), smoking status, alcohol use, evidence of reduced mobility, thrombophilia, fracture of the lower limb and family history of VTE. Socioeconomic status was defined using index of multiple deprivation (IMD), the official national measure of socioeconomic status in the UK. ${ }^{29}$ Ethnicity was extracted from the primary care record and grouped into major UK ethnic groups: white, black, Asian, mixed and others. ${ }^{30}$ BMI, smoking status and alcohol use were defined using the most recently recorded data prior to the index date. Diagnostic codes were used to define the following baseline comorbidities: hypertension, hyperlipidaemia, type 2 diabetes, peripheral vascular disease, cardiovascular disease (atrial fibrillation, angina, myocardial infarction, congestive heart failure), stroke, malignancy, chronic obstructive pulmonary disease (COPD), chronic kidney disease (CKD) (stages 3-5), liver disease and thrombophilia. Type 2 diabetes was identified using an algorithm developed for use within RCGP RSC. ${ }^{31}$ Read codes used 
to describe cardiovascular disease within RCGP RSC have been previously reported. ${ }^{30} 32$ Platelet count measures were extracted at baseline (the most recent value up to 2 years prior to the index date) and across study followup.

We examined the following medications commonly used for the management of IMIDs: non-steroidal antiinflammatory drugs (NSAIDs), oral corticosteroids, non-biologic immunosuppressant medications and biologic therapies recorded in primary care. We also examined hormone therapy (hormonal contraceptives, hormone replacement therapy (HRT)), antiplatelet agents (aspirin or ADP receptor inhibitors), warfarin, direct oral anticoagulants (DOACs), and statins. Hormonal contraceptives comprised only combined oestrogen and progestogen preparations; progesterone only contraceptives were not included as these preparations are not associated with VTE. ${ }^{33}$ HRT comprised systemic oestrogen only preparations. Active prescribing was defined as an issued prescription in the 3 months preceding and/or 1 month after the index date.

\section{Statistical analyses}

We estimated the risk of VTE, the primary outcome, using unadjusted Cox proportional hazards models, stratified by matched set (exposed cohort vs unexposed cohort), to provide overall HRs with 95\% CI for the association. Models were subsequently adjusted for all sociodemographic, clinical and VTE risk factors, as described above, in multivariable analysis. We then repeated the same analyses for PE and DVT as separate endpoints and each condition (UC, CD, RA and PsA) separately. Proportional hazards assumptions for each model were checked graphically by plotting Schoenfeld residuals.

\section{VTE risk factors}

We used multivariable Cox models to examine the influence of baseline-recorded characteristics and VTE risk factors on risk of VTE. Models were run separately in cohorts with and without an IMID. If baseline platelet count was significant in the model, we proposed to explore the impact of changing platelet count over study follow-up on VTE risk in time-updated analysis, by including platelet count as a time-updated exposure in unadjusted and multivariable-adjusted Cox models. Platelet count was both categorised as low $\left(<150 \times 10^{9} / \mathrm{L}\right)$, normal $\left(150-400 \times 10^{9} / \mathrm{L}\right)$ or high $\left(>400 \times 10^{9} / \mathrm{L}\right)$, and analysed continuously using a restricted cubic spline prespecified with 3 knots.

\section{Sensitivity analysis}

Sensitivity to the introduction of the Quality and Outcomes Framework (QOF), an incentivised programme to monitor clinical and health improvement indicators for general practice that rewards completeness of electronic coding, ${ }^{34}$ was tested by repeating the main analyses with the study follow-up beginning on January 1, 2004. All statistical analyses used R (version 3.4.1).

\section{RESULTS}

\section{Study population}

A total of 53378 people with an IMID were included, of whom $14182(26 \%)$ had a first diagnosis of UC, 9489 (18\%) CD, 23410 (44\%) RA and $6297(12 \%)$ PsA (table 1). Matched controls comprised 213512 people without an IMID of interest. Average study follow-up was 8.2 (SD 6.2) years.

\section{Baseline characteristics}

People with an IMID were similar in characteristics to their matched counterparts (table 1). Several comorbidities were more common in the exposed group including type 2 diabetes, COPD and chronic liver disease. BMI was similar although differences were observed between individuals with an IMID; more people with PsA were obese $(32.9 \%)$ than people with UC $(16.7 \%)$ or CD $(14.6 \%)$, and more people with $\mathrm{CD}$ were underweight $(5.6 \%)$ compared with other IMIDs (range 1.0-2.6\%). Use of NSAIDs, corticosteroids and immunosuppressive medications were, as expected, considerably higher in the IMID group.

\section{Risk of VTE}

Unadjusted VTE event rates were higher in the IMID group (34.9 [95\% CI 33.2 to 36.7] per 10000 personyears) compared with controls $(21.7$ [95\% CI 21.0 to 22.4] per 10000 person-years, $\mathrm{p}<0.001$ ) (figure 1); 1532 (2.9\%) people with an IMID developed VTE compared with $3804(1.8 \%)$ controls. Table 2 reported study followup and outcome events for the primary VTE outcome and the secondary outcomes of PE and DVT.

In the primary outcome analysis, adjusted models demonstrated an association between UC, CD and RA and the development of VTE, with the strength of association greatest for people with CD. Associations were consistent in analyses of separate PE and DVT endpoints (table 2, online supplemental figure 1). For PsA, a significant increase in risk was seen only for the DVT endpoint (table 2). Sensitivity analysis exploring the impact of QOF demonstrated primary results were consistent with follow-up beginning in 2004 (online supple mental table 1).

Table 3 shows associations between record characteristics and risk of developing VTE in people with an IMID and matched controls. Increasing age at entry, being overweight/obese and thrombophilia history were associated with an increased risk of VTE in both groups. Associations between VTE risk factors and risk of VTE differed, with male sex, history of fracture, current smoking and alcohol abstinence associated with an increased VTE risk only in the IMID group. Reduced mobility (increased risk) and Asian ethnicity (decreased risk) were risk factors only in controls. COPD, chronic liver 
Table 1 Covariate summary statistics for individuals with and without an immune-mediated inflammatory disease (IMID)

\begin{tabular}{|c|c|c|c|c|c|c|}
\hline & $\begin{array}{l}\text { Without } \\
\text { IMID } \\
n=213512\end{array}$ & $\begin{array}{l}\text { With } \\
\text { IMID } \\
n=53378\end{array}$ & $\begin{array}{l}\text { Ulcerative } \\
\text { colitis } \\
n=14182\end{array}$ & $\begin{array}{l}\text { Crohn's } \\
\text { disease } \\
n=9489\end{array}$ & $\begin{array}{l}\text { Psoriatic } \\
\text { arthritis } \\
n=6297\end{array}$ & $\begin{array}{l}\text { Rheumatoid } \\
\text { arthritis } \\
n=23410\end{array}$ \\
\hline & \multicolumn{6}{|c|}{ Sociodemographic characteristics } \\
\hline $\begin{array}{l}\text { Age at study entry (years) } \\
\text { Mean (SD) }\end{array}$ & $51.7(17.8)$ & $\begin{array}{l}51.6 \\
(17.4)\end{array}$ & $47.2(17.0)$ & $41.8(16.6)$ & $49.2(13.8)$ & $59.0(15.5)$ \\
\hline Male sex (n (\%)) & $\begin{array}{l}85383 \\
(40.0)\end{array}$ & $\begin{array}{l}21291 \\
(39.9)\end{array}$ & $7126(50.2)$ & 4296 (45.3) & $3093(49.1)$ & $6776(28.9)$ \\
\hline $\begin{array}{l}\text { Time since GP practice registration } \\
\text { (years). Mean (SD) }\end{array}$ & $9.1(12.1)$ & $9.1(12.3)$ & $7.8(11.1)$ & $7.5(10.6)$ & $9.2(11.6)$ & $10.4(13.5)$ \\
\hline \multicolumn{7}{|l|}{ Ethnicity (n (\%)) } \\
\hline Asian & $9569(5.8)$ & $\begin{array}{l}2434 \\
(5.7)\end{array}$ & $724(6.5)$ & $347(4.7)$ & $249(4.9)$ & $1114(5.9)$ \\
\hline Black & $4121(2.5)$ & $643(1.5)$ & $127(1.1)$ & $91(1.2)$ & $22(0.4)$ & $403(2.1)$ \\
\hline Mixed & $1483(0.9)$ & $346(0.8)$ & $85(0.8)$ & $67(0.9)$ & $44(0.9)$ & $150(0.8)$ \\
\hline Other & $1448(0.9)$ & $296(0.7)$ & $93(0.8)$ & $54(0.7)$ & $26(0.5)$ & $123(0.7)$ \\
\hline White & $\begin{array}{l}148832 \\
(90.0)\end{array}$ & $\begin{array}{l}38708 \\
(91.2)\end{array}$ & $10099(90.8)$ & $6763(92.4)$ & 4727 (93.3) & $17119(90.5)$ \\
\hline Missing & $\begin{array}{l}48059 \\
(22.5)\end{array}$ & $\begin{array}{l}10951 \\
(20.5)\end{array}$ & $3054(21.5)$ & $2167(22.8)$ & $1229(19.5)$ & 4501 (19.2) \\
\hline \multicolumn{7}{|c|}{ Index of multiple deprivation quintile ( $(\%))$} \\
\hline 1 (most deprived) & $\begin{array}{l}29144 \\
(13.6)\end{array}$ & $\begin{array}{l}7293 \\
(13.7)\end{array}$ & $1689(11.9)$ & $1324(14.0)$ & $803(12.8)$ & 3477 (14.9) \\
\hline 2 & $\begin{array}{l}32323 \\
(15.1)\end{array}$ & $\begin{array}{l}8274 \\
(15.5)\end{array}$ & $2109(14.9)$ & $1518(16.0)$ & $919(14.6)$ & $3728(15.9)$ \\
\hline 3 & $\begin{array}{l}41379 \\
(19.4)\end{array}$ & $\begin{array}{l}10570 \\
(19.8)\end{array}$ & $2680(18.9)$ & $1901(20.0)$ & $1250(19.9)$ & 4739 (20.2) \\
\hline 4 & $\begin{array}{l}50087 \\
(23.5)\end{array}$ & $\begin{array}{l}12439 \\
(23.3)\end{array}$ & 3475 (24.5) & $2186(23.0)$ & $1452(23.1)$ & $5326(22.8)$ \\
\hline 5 (least deprived) & $\begin{array}{l}56209 \\
(26.3)\end{array}$ & $\begin{array}{l}13684 \\
(25.6)\end{array}$ & $3963(27.9)$ & $2350(24.8)$ & $1720(27.3)$ & $5651(24.1)$ \\
\hline IMD not recorded & $4370(2.0)$ & $\begin{array}{l}1118 \\
(2.1)\end{array}$ & $266(1.9)$ & $210(2.2)$ & $153(2.4)$ & $489(2.1)$ \\
\hline \multicolumn{7}{|l|}{ VTE risk factors (n (\%)) } \\
\hline \multicolumn{7}{|l|}{$\mathrm{BMI}\left(\mathrm{kg} / \mathrm{m}^{2}\right)$} \\
\hline Underweight ( $\leq 18.5)$ & $4704(2.2)$ & $\begin{array}{l}1571 \\
(2.9)\end{array}$ & $368(2.6)$ & $536(5.6)$ & $60(1.0)$ & $607(2.6)$ \\
\hline Normal weight (18.5-25) & $\begin{array}{l}73675 \\
(34.5)\end{array}$ & $\begin{array}{l}19280 \\
(36.1)\end{array}$ & $5721(40.3)$ & 4225 (44.5) & $1576(25.0)$ & $7758(33.1)$ \\
\hline Overweight (25-30) & $\begin{array}{l}67076 \\
(31.4)\end{array}$ & $\begin{array}{l}16664 \\
(31.2)\end{array}$ & $4352(30.7)$ & $2446(25.8)$ & $2131(33.8)$ & 7735 (33.0) \\
\hline Obese $(\geq 30)$ & $\begin{array}{l}44303 \\
(20.7)\end{array}$ & $\begin{array}{l}11611 \\
(21.8)\end{array}$ & $2367(16.7)$ & $1386(14.6)$ & 2071 (32.9) & $5787(24.7)$ \\
\hline BMI not recorded & $\begin{array}{l}23754 \\
(11.1)\end{array}$ & $\begin{array}{l}4252 \\
(8.0)\end{array}$ & $1374(9.7)$ & $896(9.4)$ & 459 ( 7.3) & $1523(6.5)$ \\
\hline \multicolumn{7}{|l|}{ Smoking status } \\
\hline Non-smoker & $\begin{array}{l}94985 \\
(44.5)\end{array}$ & $\begin{array}{l}21620 \\
(40.5)\end{array}$ & $6328(44.6)$ & 3917 (41.3) & $2522(40.1)$ & $8853(37.8)$ \\
\hline Current smoker & $\begin{array}{l}52035 \\
(24.4)\end{array}$ & $\begin{array}{l}13070 \\
(24.5)\end{array}$ & $2574(18.1)$ & $2914(30.7)$ & $1519(24.1)$ & $6063(25.9)$ \\
\hline Ex-smoker & $\begin{array}{l}63798 \\
(29.9)\end{array}$ & $\begin{array}{l}18315 \\
(34.3)\end{array}$ & $5147(36.3)$ & $2551(26.9)$ & $2232(35.4)$ & 8385 (35.8) \\
\hline Smoking status not recorded & $2694(1.3)$ & $373(0.7)$ & $133(0.9)$ & $107(1.1)$ & $24(0.4)$ & $109(0.5)$ \\
\hline
\end{tabular}


Table 1 Continued

\begin{tabular}{|c|c|c|c|c|c|c|}
\hline & $\begin{array}{l}\text { Without } \\
\text { IMID } \\
\mathrm{n}=213512\end{array}$ & $\begin{array}{l}\text { With } \\
\text { IMID } \\
n=53378\end{array}$ & $\begin{array}{l}\text { Ulcerative } \\
\text { colitis } \\
n=14182\end{array}$ & $\begin{array}{l}\text { Crohn's } \\
\text { disease } \\
n=9489\end{array}$ & $\begin{array}{l}\text { Psoriatic } \\
\text { arthritis } \\
n=6297\end{array}$ & $\begin{array}{l}\text { Rheumatoid } \\
\text { arthritis } \\
n=23410\end{array}$ \\
\hline & \multicolumn{6}{|c|}{ Sociodemographic characteristics } \\
\hline Non-drinker & $\begin{array}{l}36623 \\
(17.2)\end{array}$ & $\begin{array}{l}10608 \\
(19.9)\end{array}$ & $2371(16.7)$ & $1798(18.9)$ & 1035 (16.4) & $5404(23.1)$ \\
\hline Within limits & $\begin{array}{l}117939 \\
(55.2)\end{array}$ & $\begin{array}{l}29316 \\
(54.9)\end{array}$ & 7727 (54.5) & $4917(51.8)$ & $3508(55.7)$ & $13164(56.2)$ \\
\hline Over recommended I imits & $\begin{array}{l}30096 \\
(14.1)\end{array}$ & $\begin{array}{l}7145 \\
(13.4)\end{array}$ & $2083(14.7)$ & $1228(12.9)$ & $1067(16.9)$ & $2767(11.8)$ \\
\hline Alcoholism & $3438(1.6)$ & $823(1.5)$ & $217(1.5)$ & $138(1.5)$ & $128(2.0)$ & $340(1.5)$ \\
\hline Alcohol intake not recorded & $\begin{array}{l}25416 \\
(11.9)\end{array}$ & $\begin{array}{l}5486 \\
(10.3)\end{array}$ & $1784(12.6)$ & $1408(14.8)$ & $559(8.9)$ & 1735 ( 7.4$)$ \\
\hline Reduced mobility & $3562(1.7)$ & $\begin{array}{l}1022 \\
(1.9)\end{array}$ & $184(1.3)$ & $117.2(1.2)$ & $85(1.3)$ & $636(2.7)$ \\
\hline Thrombophilia & $151(0.1)$ & $49(0.1)$ & $11(0.1)$ & $11(0.1)$ & $7(0.1)$ & $20(0.1)$ \\
\hline Family history of VTE & $403(0.2)$ & $113(0.2)$ & $28(0.2)$ & $27(0.3)$ & $21(0.3)$ & $37(0.2)$ \\
\hline History of fracture & $\begin{array}{l}14542 \\
(6.8)\end{array}$ & $\begin{array}{l}3887 \\
(7.3)\end{array}$ & $978(6.9)$ & $593(6.2)$ & $467(7.4)$ & $1849(7.9)$ \\
\hline \multicolumn{7}{|l|}{ Platelet count category (n (\%)) } \\
\hline $\operatorname{Low}\left(<150 \times 10^{9} / \mathrm{L}\right)$ & $2393(1.1)$ & $635(1.2)$ & $141(1.0)$ & $100(1.1)$ & $85(1.3)$ & 309 (1.3) \\
\hline Normal (150-400×10\%/L) & $\begin{array}{l}83707 \\
(39.2)\end{array}$ & $\begin{array}{l}29655 \\
(55.6)\end{array}$ & $7251(51.1)$ & $4610(48.6)$ & $3910(62.1)$ & $13884(59.3)$ \\
\hline High $\left(>400 \times 10^{9} / L\right)$ & $3111(1.5)$ & $\begin{array}{l}4204 \\
(7.9)\end{array}$ & $908(6.4)$ & $1158(12.2)$ & $293(4.7)$ & $1845(7.9)$ \\
\hline Missing & $\begin{array}{l}124301 \\
(58.2)\end{array}$ & $\begin{array}{l}18884 \\
(35.4)\end{array}$ & $5882(41.5)$ & $3621(38.2)$ & 2009 (31.9) & $7372(31.5)$ \\
\hline \multicolumn{7}{|l|}{ Comorbidity (n (\%)) } \\
\hline Hypertension & $\begin{array}{l}43296 \\
(20.3)\end{array}$ & $\begin{array}{l}11298 \\
(21.2)\end{array}$ & 2206 (15.2) & $1043(10.7)$ & $1334(21.2)$ & $6809(29.1)$ \\
\hline Hyperlipidaemia & $\begin{array}{l}51377 \\
(24.1)\end{array}$ & $\begin{array}{l}12241 \\
(22.9)\end{array}$ & 2606 (18.4) & $1243(13.1)$ & $1542(24.5)$ & $6850(29.3)$ \\
\hline Type 2 diabetes & $\begin{array}{l}12423 \\
(5.8)\end{array}$ & $\begin{array}{l}3466 \\
(6.5)\end{array}$ & $714(5.0)$ & 307 (3.2) & $452(7.2)$ & $1993(8.5)$ \\
\hline Peripheral vascular disease & $1948(0.9)$ & $530(1.0)$ & $98(0.7)$ & $61(0.6)$ & $59(0.9)$ & $312(1.3)$ \\
\hline Atrial fibrillation & $4569(2.1)$ & $\begin{array}{l}1227 \\
(2.3)\end{array}$ & $251(1.8)$ & $118(1.2)$ & $79(1.3)$ & 779 (3.3) \\
\hline Myocardial infarction & $4325(2.0)$ & $\begin{array}{l}1280 \\
(2.4)\end{array}$ & $286(2.0)$ & $125(1.3)$ & $102(1.6)$ & 767 (3.3) \\
\hline Stroke & $3344(1.6)$ & $818(1.5)$ & $164(1.2)$ & $99(1.0)$ & $66(1.0)$ & $489(2.1)$ \\
\hline Heart failure & $2276(1.1)$ & $654(1.2)$ & $129(0.9)$ & $57(0.6)$ & $39(0.6)$ & $429(1.8)$ \\
\hline Chronic kidney disease stages $3-5$ & $6936(3.2)$ & $\begin{array}{l}1819 \\
(3.4)\end{array}$ & $294(2.1)$ & $168(1.8)$ & $131(2.1)$ & $1226(5.2)$ \\
\hline $\begin{array}{l}\text { Chronic obstructive pulmonary } \\
\text { disease }\end{array}$ & $5628(2.6)$ & $\begin{array}{l}2039 \\
(3.8)\end{array}$ & $359(2.5)$ & $225(2.4)$ & $130(2.1)$ & $1325(5.7)$ \\
\hline Chronic liver disease & $992(0.5)$ & $559(1.0)$ & $208(1.5)$ & $87(0.9)$ & $61(1.0)$ & $203(0.9)$ \\
\hline Malignancy & $8703(4.1)$ & $\begin{array}{l}2169 \\
(4.1)\end{array}$ & 455 (3.2) & $238(2.5)$ & $211(3.4)$ & $1265(5.4)$ \\
\hline \multicolumn{7}{|l|}{ Medication use (n (\%)) } \\
\hline NSAID use & $\begin{array}{l}49829 \\
(23.3)\end{array}$ & $\begin{array}{l}20385 \\
(38.2)\end{array}$ & $2621(18.5)$ & 1754 (18.5) & $3509(55.7)$ & $12501(53.4)$ \\
\hline Corticosteroid use & $\begin{array}{l}10438 \\
(4.9)\end{array}$ & $\begin{array}{l}13166 \\
(24.7)\end{array}$ & $3283(23.1)$ & $2734(28.8)$ & $893(14.2)$ & $6256(26.7)$ \\
\hline
\end{tabular}


Table 1 Continued

\begin{tabular}{|c|c|c|c|c|c|c|}
\hline & $\begin{array}{l}\text { Without } \\
\text { IMID } \\
\text { n=213 } 512\end{array}$ & $\begin{array}{l}\text { With } \\
\text { IMID } \\
n=53378\end{array}$ & $\begin{array}{l}\text { Ulcerative } \\
\text { colitis } \\
n=14182\end{array}$ & $\begin{array}{l}\text { Crohn's } \\
\text { disease } \\
\mathrm{n}=9489\end{array}$ & $\begin{array}{l}\text { Psoriatic } \\
\text { arthritis } \\
n=6297\end{array}$ & $\begin{array}{l}\text { Rheumatoid } \\
\text { arthritis } \\
n=23410\end{array}$ \\
\hline & \multicolumn{6}{|c|}{ Sociodemographic characteristics } \\
\hline $\begin{array}{l}\text { Immunosuppressive medication (in } \\
\text { primary care) }\end{array}$ & $1654(0.8)$ & $\begin{array}{l}18248 \\
(34.2)\end{array}$ & $1830(12.9)$ & $2338(24.6)$ & $2801(44.5)$ & $11279(48.2)$ \\
\hline Statin use & $\begin{array}{l}29735 \\
(13.9)\end{array}$ & $\begin{array}{l}7655 \\
(14.3)\end{array}$ & $1378(9.7)$ & $674(7.1)$ & $839(13.3)$ & $4764(20.4)$ \\
\hline Antiplatelet therapy & $\begin{array}{l}17620 \\
(8.3)\end{array}$ & $\begin{array}{l}4484 \\
(8.4)\end{array}$ & $871(6.1)$ & $413(4.4)$ & $416(6.6)$ & $2784(11.9)$ \\
\hline Warfarin & $2842(1.3)$ & $760(1.4)$ & $150(1.1)$ & $72(0.8)$ & $49(0.8)$ & $489(2.1)$ \\
\hline Direct oral anticoagulants & $939(0.4)$ & $288(0.5)$ & $49(0.3)$ & $25(0.3)$ & $21(0.3)$ & $193(0.8)$ \\
\hline Hormone replacement therapy & $4283(2.0)$ & $\begin{array}{l}1359 \\
(2.5)\end{array}$ & $204(1.4)$ & $196(2.1)$ & $206(3.3)$ & $753(3.2)$ \\
\hline Oral contraceptive use & $9681(4.5)$ & $\begin{array}{l}2434 \\
(4.6)\end{array}$ & $856(6.0)$ & $817(8.6)$ & $215(3.4)$ & $546(2.3)$ \\
\hline
\end{tabular}

BMI, body mass index; NSAID, non-steroidal anti-inflammatory drug; VTE, venous thromboembolism.

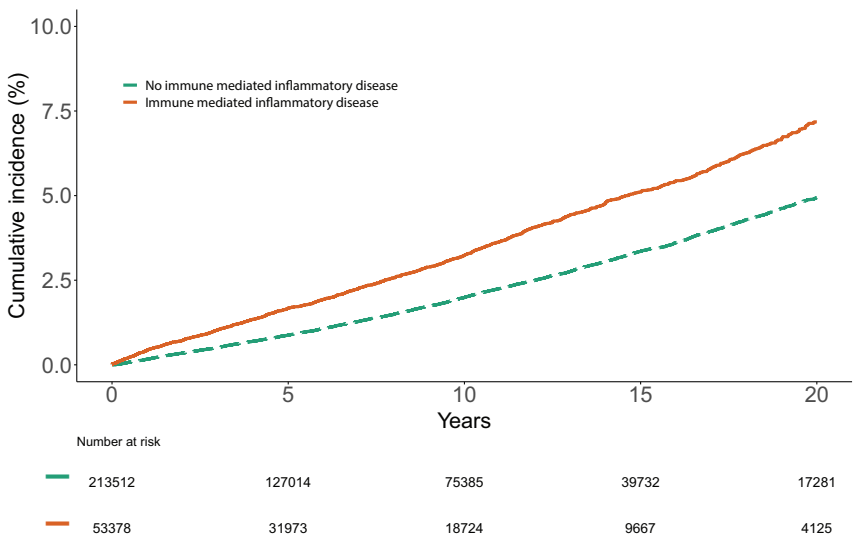

Figure 1 Cumulative incidence of VTE in individuals with an immune-mediated inflammatory diseases compared with matched controls. Individuals with both DVT and PE on the same day $(n=180)$ classified as having had a PE. DVT, deep vein thrombosis; PE, pulmonary embolism; VTE, venous thromboembolism.

disease, peripheral vascular disease and family history of VTE were all associated with an increased risk of VTE only in controls. CKD was associated with increased risk of VTE only in people with an IMID. For medication, warfarin, DOACs, corticosteroids, and, in females, use of oral contraceptives, were associated with an increased risk of VTE in both groups. Statins (decreased risk) and NSAIDs (increased risk) were risk factors only in the IMID group. Risk of VTE was increased in people with an IMID and low number of platelets $\left(<150 \times 10^{9} / \mathrm{L}\right)$, and in people without an IMID and high number of platelets $\left(>400 \times 10^{9} / \mathrm{L}\right)$. In the IMID group, relative to people with $\mathrm{UC}$, risk of VTE was increased in CD only.
Association of platelet count across study follow-up with risk of VTE

To further interrogate the relationship between baseline platelets and VTE in each group, a time-updated analysis was undertaken. Individuals with at least one platelet count were included in the analysis (96\% of those with an IMID and $75 \%$ of those without an IMID). Platelet count across study follow-up was initially categorised as low, normal or high (table 4). High and low platelet counts were more common in individuals with an IMID (proportion of individuals with $1+$ one high platelet count $29.2 \%$, low count $10.9 \%$ ) compared with those without an IMID (high count $11.5 \%$, low count $7.4 \%$ ).

Higher time-varying platelet counts were associated with an increased risk of VTE in individuals with and without an IMID (table 4, figure 1). Figure 2 confirms the association between time-updated lower and higher platelet count and higher risk of VTE in both groups when modelling platelet count as a non-linear continuous variable; a positive association was also seen for platelet counts $<200 \times 10^{9} / \mathrm{L}$.

\section{DISCUSSION}

Our study shows that VTE is more common in people with UC, CD, RA, and PsA compared with people without these IMIDs. UC, CD and RA were independently associated to a similar degree with increased risk of VTE, while the wide CI for PSA suggests we lacked statistical power to detect a difference in this group. Established risk factors for VTE had a similar prevalence in people with an IMID compared with the wider population, and similar strengths of association were observed in people with and without an IMID for higher age, being overweight or obese, thrombophilia, malignancy, and corticosteroid use. Notable differences were also observed; only in people with an IMID did we find evidence that 
Table 2 Associations between immune-mediated inflammatory diseases (IMID) and risk of VTE in unadjusted and multivariable analysis

HR (95\% Cl)

No. Patient years at risk Events Unadjusted Adjusted

Primary outcome: risk of VTE

All immune mediated inflammatory diseases

\begin{tabular}{|c|c|c|c|c|c|}
\hline Controls & 213512 & 1756381 & 3804 & 1.00 (ref) & 1.00 (ref) \\
\hline Immune mediated inflammatory disease & 53378 & 438743 & 1532 & $1.62(1.52,1.71)$ & $1.46(1.36,1.56)$ \\
\hline \multicolumn{6}{|l|}{ Ulcerative colitis } \\
\hline Controls & 56728 & 476506 & 956 & 1.00 (ref) & 1.00 (ref) \\
\hline Ulcerative colitis & 14182 & 119635 & 335 & $1.40(1.23,1.58)$ & $1.27(1.10,1.45)$ \\
\hline \multicolumn{6}{|l|}{ Crohn's disease } \\
\hline Controls & 37956 & 307373 & 460 & 1.00 (ref) & 1.00 (ref) \\
\hline Crohn's disease & 9489 & 76685 & 220 & $1.92(1.63,2.25)$ & $1.74(1.45,2.08)$ \\
\hline \multicolumn{6}{|l|}{ Rheumatoid arthritis } \\
\hline Controls & 93640 & 770424 & 2020 & 1.00 (ref) & 1.00 (ref) \\
\hline Rheumatoid arthritis & 23410 & 19022 & 845 & $1.69(1.56,1.83)$ & $1.54(1.40,1.69)$ \\
\hline \multicolumn{6}{|l|}{ Psoriatic arthritis } \\
\hline Controls & 25188 & 202078 & 368 & 1.00 (ref) & 1.00 (ref) \\
\hline All Immune mediated inflammatory diseases & 6297 & 51400 & 132 & $1.41(1.16,1.72)$ & $1.20(0.96,1.52)$ \\
\hline \multicolumn{6}{|l|}{ Secondary outcome: risk of PE } \\
\hline \multicolumn{6}{|l|}{ All Immune mediated inflammatory diseases } \\
\hline Controls & 213509 & 1777837 & 1737 & 1.00 (ref) & 1.00 (ref) \\
\hline Immune mediated inflammatory disease & 53370 & 443470 & 672 & $1.57(1.44,1.72)$ & $1.43(1.29,1.58)$ \\
\hline \multicolumn{6}{|l|}{ Ulcerative colitis } \\
\hline Controls & 56728 & 482186 & 452 & 1.00 (ref) & 1.00 (ref) \\
\hline Ulcerative colitis & 14182 & 120710 & 149 & $1.35(1.12,1.62)$ & $1.23(1.01,1.49)$ \\
\hline \multicolumn{6}{|l|}{ Crohn's disease } \\
\hline Controls & 37956 & 310470 & 207 & 1.00 (ref) & 1.00 (ref) \\
\hline Crohn's disease & 9489 & 77393 & 98 & $1.96(1.55,2.49)$ & $1.69(1.29,2.20)$ \\
\hline \multicolumn{6}{|l|}{ Rheumatoid arthritis } \\
\hline Controls & 93639 & 780883 & 916 & 1.00 (ref) & 1.00 (ref) \\
\hline Rheumatoid arthritis & 23408 & 193534 & 373 & $1.66(1.47,1.87)$ & $1.57(1.36,1.80)$ \\
\hline \multicolumn{6}{|l|}{ Psoriatic arthritis } \\
\hline Controls & 25186 & 204299 & 161 & 1.00 (ref) & 1.00 (ref) \\
\hline Psoriatic arthritis & 6297 & 51833 & 52 & $1.27(0.93,1.73)$ & $1.08(0.75,1.55)$ \\
\hline \multicolumn{6}{|l|}{ Secondary outcome: risk of DVT } \\
\hline \multicolumn{6}{|l|}{ All Immune mediated inflammatory diseases } \\
\hline Controls & 213510 & 1773186 & 2335 & 1.00 (ref) & 1.00 (ref) \\
\hline Immune mediated inflammatory disease & 53372 & 441330 & 978 & $1.70(1.58,1.83)$ & $1.57(1.45,1.71)$ \\
\hline \multicolumn{6}{|l|}{ Ulcerative colitis } \\
\hline Controls & 56728 & 480944 & 583 & 1.00 (ref) & 1.00 (ref) \\
\hline Ulcerative colitis & 14182 & 120309 & 207 & $1.43(1.22,1.68)$ & $1.33(1.13,1.57)$ \\
\hline \multicolumn{6}{|l|}{ Crohn's disease } \\
\hline Controls & 37956 & 309769 & 279 & 1.00 (ref) & 1.00 (ref) \\
\hline Crohn's disease & 9489 & 77115 & 140 & $2.05(1.67,2.50)$ & $1.96(1.57,2.45)$ \\
\hline Rheumatoid arthritis & & & & RA & \\
\hline Controls & 93640 & 778523 & 1242 & 1.00 (ref) & 1.00 (ref) \\
\hline Rheumatoid arthritis & 23408 & 192276 & 542 & $1.78(1.61,1.97)$ & $1.64(1.45,1.84)$ \\
\hline \multicolumn{6}{|l|}{ Psoriatic arthritis } \\
\hline Controls & 25817 & 203949 & 231 & 1.00 (ref) & 1.00 (ref) \\
\hline Psoriatic arthritis & 6297 & 51630 & 89 & $1.52(1.21,1.97)$ & $1.34(1.01,1.77)$ \\
\hline
\end{tabular}

Adjusted for age, sex, IMD quintile, ethnicity, BMI category, smoking status, alcohol use category, hypertension, hyperlipidaemia, type 2 diabetes, peripheral arterial disease, atrial fibrillation, myocardial infarction, stroke, heart failure, CKD stage 3-5, COPD, chronic liver disease, malignancy, reduced mobility, use of NSAIDs, antiplatelets, warfarin, DOACs, hormone replacement therapy, oestrogen contraceptives, immunotherapy, corticosteroids, statins and baseline platelet category.

BMI, body mass index; CKD, chronic kidney disease; COPD, chronic obstructive pulmonary disease; DVT, deep vein thrombosis; DOAC, direct oral anticoagulants; NSAID, non-steroidal anti-inflammatory drug; PE, pulmonary embolism; RA, rheumatoid arthritis 
Table 3 Association of baseline recorded characteristics and VTE risk factors with risk of VTE among individuals with and without immune-mediated inflammatory diseases (IMID) in multivariable analysis

\begin{tabular}{|c|c|c|}
\hline & $\begin{array}{l}\text { Without IMID } \\
(\mathrm{n}=213512)\end{array}$ & $\begin{array}{l}\text { With IMID } \\
(n=53378)\end{array}$ \\
\hline \multicolumn{3}{|l|}{ Type of IMID } \\
\hline Ulcerative colitis & NA & 1.00 (ref) \\
\hline Crohn's disease & NA & $1.20(1.01,1.42)$ \\
\hline Psoriatic arthritis & NA & $0.87(0.71,1.07)$ \\
\hline Rheumatoid arthritis & NA & $1.11(0.97,1.28)$ \\
\hline \multicolumn{3}{|c|}{ Sociodemographic characteristics } \\
\hline $\begin{array}{l}\text { Age at study entry } \\
\text { (years) }\end{array}$ & $1.04(1.04,1.05)$ & $1.03(1.03,1.04)$ \\
\hline Male sex & $1.02(0.95,1.10)$ & $1.13(1.01,1.26)$ \\
\hline \multicolumn{3}{|l|}{ Ethnicity } \\
\hline Asian & $0.46(0.34,0.63)$ & $0.96(0.70,1.31)$ \\
\hline Black & $1.19(0.90,1.56)$ & $1.08(0.62,1.87)$ \\
\hline Mixed & $1.40(0.87,2.27)$ & $1.01(0.45,2.27)$ \\
\hline Other & $0.96(0.51,1.78)$ & $0.45(0.11,1.80)$ \\
\hline Missing & $1.04(0.96,1.12)$ & $0.96(0.84,1.09)$ \\
\hline White & 1.00 (ref) & 1.00 (ref) \\
\hline \multicolumn{3}{|c|}{ Index of multiple deprivation quintile (IMD) } \\
\hline 1 (most deprived) & 1.00 (ref) & 1.00 (ref) \\
\hline 2 & $1.05(0.94,1.19)$ & $1.02(0.84,1.22)$ \\
\hline 3 & $0.97(0.86,1.09)$ & $0.95(0.79,1.13)$ \\
\hline 4 & $0.93(0.83,1.03)$ & $0.94(0.79,1.12)$ \\
\hline 5 (least deprived) & $0.94(0.84,1.04)$ & $0.86(0.72,1.02)$ \\
\hline IMD not recorded & $0.87(0.67,1.12)$ & $1.00(0.69,1.45)$ \\
\hline \multicolumn{3}{|l|}{ VTE risk factors } \\
\hline \multicolumn{3}{|l|}{ BMI $\left(\mathrm{kg} / \mathrm{m}^{2}\right)$} \\
\hline Underweight $(\leq 18.5)$ & $1.06(0.80,1.41)$ & $0.99(0.68,1.44)$ \\
\hline $\begin{array}{l}\text { Normal weight } \\
(18.5-25)\end{array}$ & 1.00 (ref) & 1.00 (ref) \\
\hline Overweight (25-30) & $1.24(1.14,1.35)$ & $1.23(1.08,1.39)$ \\
\hline Obese $(\geq 30)$ & $1.91(1.75,2.08)$ & $1.66(1.45,1.91)$ \\
\hline BMI not recorded & $1.14(0.98,1.32)$ & $1.31(1.03,1.65)$ \\
\hline \multicolumn{3}{|l|}{ Smoking status } \\
\hline Non-smoker & 1.00 (ref) & 1.00 (ref) \\
\hline Current smoker & $1.08(0.99,1.17)$ & $1.22(1.07,1.39)$ \\
\hline Ex-smoker & $1.06(0.98,1.15)$ & $1.07(0.95,1.21)$ \\
\hline $\begin{array}{l}\text { Smoking status not } \\
\text { recorded }\end{array}$ & $0.32(0.12,0.87)$ & $0.37(0.05,2.62)$ \\
\hline \multicolumn{3}{|l|}{ Alcohol intake } \\
\hline Non-drinker & $1.07(0.98,1.16)$ & $1.17(1.03,1.32)$ \\
\hline Within limits & 1.00 (ref) & 1.00 (ref) \\
\hline $\begin{array}{l}\text { Over recommended } \\
\text { limits }\end{array}$ & $1.01(0.91,1.11)$ & $0.90(0.77,1.06)$ \\
\hline Alcoholism & $1.19(0.92,1.54)$ & $1.44(0.99,2.08)$ \\
\hline $\begin{array}{l}\text { Alcohol intake not } \\
\text { recorded }\end{array}$ & $1.07(0.93,1.23)$ & $1.17(0.94,1.46)$ \\
\hline Reduced mobility & $1.39(1.12,1.72)$ & $0.96(0.67,1.37)$ \\
\hline Family history of VTE & $3.10(1.60,6.01)$ & $1.29(0.32,5.19)$ \\
\hline
\end{tabular}

Continued
Table 3 Continued

$\begin{array}{ll}\begin{array}{l}\text { Without IMID } \\ (n=213512)\end{array} & \begin{array}{l}\text { With IMID } \\ (n=53378)\end{array}\end{array}$

\begin{tabular}{lll}
\hline Thrombophilia & $4.66(2.31,9.40)$ & $4.13(1.53$, \\
& & $11.11)$ \\
History of fracture & $1.11(0.98,1.25)$ & $1.29(1.08,1.55)$ \\
Platelet count & $1.16(0.90,1.50)$ & $1.23(1.01,1.50)$ \\
$\quad \begin{array}{l}\text { Normal } \\
\left(150-400 \times 10^{9} / \mathrm{L}\right)\end{array}$ & $1.00($ ref $)$ & $1.00($ ref $)$ \\
\hline High $\left(>400 \times 10^{9} / \mathrm{L}\right)$ & $1.37(1.02,1.84)$ & $1.07(0.67,1.72)$ \\
\hline Missing & $0.55(0.51,0.59)$ & $1.07(0.94,1.22)$
\end{tabular}

\section{Comorbidity}

Hypertension

$1.02(0.94,1.10) \quad 1.02(0.90,1.15)$

Hyperlipidaemia $\quad 1.03(0.94,1.15) \quad 1.08(0.91,1.28)$

Type 2 diabetes $\quad 1.03(0.90,1.17) \quad 0.90(0.72,1.11)$

Peripheral vascular $\quad 1.30(1.02,1.65) \quad 0.73(0.44,1.20)$

disease

Atrial fibrillation $\quad 0.44(0.34,0.57) \quad 0.32(0.21,0.48)$

Myocardial infarction $\quad 0.97(0.80,1.17) \quad 1.11(0.84,1.48)$

Stroke $\quad 1.15(0.93,1.43) \quad 0.91(0.62,1.34)$

Heart failure $\quad 0.99(0.76,1.29) \quad 1.10(0.76,1.61)$

Chronic kidney disease $1.16(0.98,1.37) \quad 1.29(1.00,1.67)$

stages 3 to 5

Chronic obstructive $\quad 1.35(1.14,1.59) \quad 1.21(0.96,1.53)$

pulmonary disease

Chronic liver disease $\quad 1.79(1.24,2.59) \quad 1.29(0.81,2.07)$

Malignancy $\quad 1.30(1.14,1.48) \quad 1.27(1.02,1.57)$

Medication use

NSAID use $\quad 1.26(1.15,1.38) \quad 1.05(0.92,1.21)$

Corticosteroid use $\quad 1.33(1.16,1.54) \quad 1.22(1.06,1.40)$

Immunosuppressive $\quad 1.55(1.15,2.10) \quad 1.14(0.99,1.30)$

medication use

Statin use $\quad 0.85(0.74,0.98) \quad 0.87(0.68,1.10)$

Antiplatelet therapy $\quad 0.94(0.83,1.07) \quad 0.94(0.76,1.16)$

Warfarin use $\quad 2.37(1.88,3.00) \quad 4.20(2.96,5.96)$

Direct oral $\quad 2.68(1.84,3.91) \quad 8.36(5.40$,

anticoagulants 12.94$)$

Hormone replacement $\quad 0.77(0.59,1.00) \quad 1.12(0.80,1.56)$

therapy*

Combined oral $\quad 1.20(0.87,1.66) \quad 1.63(1.10,2.40)$ contraceptive use $\dagger$

${ }^{*}$ For females only, HRs were $1.15(95 \% \mathrm{Cl} 0.83$ to 1.48$)$ in people without an IMID and 1.11 (95\% Cl 0.78 to 1.44) in people with an IMID.

†For females only, HRs were $1.85(95 \% \mathrm{Cl} 1.45$ to 2.45$)$ in people without an IMID and $1.64(95 \% \mathrm{Cl} 1.24$ to 2.04$)$ in people with an IMID. Values are HRs with $95 \%$ Cls.

BMI, body mass index; NSAID, non-steroidal anti-inflammatory drug.

male sex, current smoking, CKD, and history of fracture were independent risk factors for VTE. Across study follow-up, abnormal platelet counts were found to be independently associated with risk of VTE in both groups but were substantially more common in people with an IMID.

Our study for the first time assesses the risk of developing VTE across four common IMIDs using the same study design. VTE incidence in this study was similar to that 
Table 4 Association of time-varying platelet count with time to VTE in individuals with and without immune-mediated inflammatory diseases (IMID) in adjusted and multivariable analysis

\section{Without IMID}

$\mathrm{n}=160969$, VTE events $=3250$

\begin{tabular}{|c|c|c|c|c|}
\hline Platelets & $\begin{array}{l}\text { Unadjusted } \\
\text { HR (95\% Cl) }\end{array}$ & $\begin{array}{l}\text { Adjusted }^{*} \\
\text { HR }(95 \% \mathrm{Cl})\end{array}$ & $\begin{array}{l}\text { Unadjusted } \\
\text { HR (95\% Cl) }\end{array}$ & $\begin{array}{l}\text { Adjusted }^{*} \\
\text { HR }(95 \% \mathrm{Cl})\end{array}$ \\
\hline Low $\left(<150 \times 10^{9} / L\right)$ & $1.88(1.60-2.20)$ & $1.06(0.98-1.14)$ & $1.62(1.23-2.12)$ & $1.24(0.94-1.62)$ \\
\hline Normal (150-400×109/L) & 1.00 (ref) & 1.00 (ref) & 1.00 (ref) & 1.00 (ref) \\
\hline $\operatorname{High}\left(>400 \times 10^{9} / L\right)^{*}$ & $2.13(1.83-2.43)$ & $1.98(1.73-2.26)$ & $1.59(1.35-1.87)$ & $1.72(1.46-2.03)$ \\
\hline
\end{tabular}

${ }^{*}$ Adjusted for age, sex, index of multiple deprivation quintile, ethnicity, body mass index category, smoking status, alcohol category, hypertension, hyperlipidaemia, type 2 diabetes, peripheral arterial disease, atrial fibrillation, myocardial infarction, stroke, heart failure, Chronic kidney disease stage 3-5, Chronic obstructive pulmonary disease, chronic liver disease, malignancy, reduced mobility, use of medication (NSAIDs, antiplatelets, warfarin, DOACs, hormone replacement therapy, oestrogen contraceptives, immunotherapy, corticosteroids and statins). Individuals with at least one valid platelet measure over the study period included.

DOAC, direct oral anticoagulants; ,NSAID non-steroidal anti-inflammatory drug.

previously reported in the $\mathrm{UK},{ }^{1}$ and a similar difference in incidence rates between people with PsA, RA and psoriasis and matched controls was recently reported using UK primary care data. ${ }^{6}$ Results are in keeping with previous studies that have consistently found people with RA to be at increased risk of VTE. ${ }^{5} 6935$ Ogdie et al conducted the first observational study of VTE risk in people with PsA and similar to our study, likely lacked power to detect a difference for overall VTE risk, ${ }^{6}$ suggesting further evaluation of VTE risk in patients with PsA in even larger cohorts is an important area for future research.

We also demonstrate an interesting u-shaped association between platelet count and VTE risk, with both high and low platelet count demonstrated to be markers of increased risk compared with normal platelet count in people with IMIDs managed in primary care. Given initial positive associations with baseline platelet count, and the recognised interaction between inflammatory cytokines and platelet function, ${ }^{36}$ we explored this association in depth using time-updated platelet counts across study follow-up to further delineate thresholds of risk/association with time to VTE. Time-updated high and low platelets were independently associated with risk of VTE in both people with and without an IMID; however, high and low platelet counts were much more common in people with an IMID, suggesting particular clinical utility in this group. Although the direction of effects was the same for the baseline and time-varying platelet analysis, differences in statistical significance and effect size may relate to the increased power and greater predictive ability gained from incorporating time-updated platelet measures. Our findings for high platelet count are in keeping with previous studies that have demonstrated thrombocytosis to be both a risk factor for VTE in inpatient populations, ${ }^{12}$ and to be associated with increased mortality risk in population-based cohorts. ${ }^{37}$ To our knowledge, the association between low platelet count and increased VTE risk is novel, with one possible explanation that clumping of platelets occurs with platelet activation and could cause an artificially low platelet count.

Our evaluation of VTE risk factors is in keeping with other less comprehensive previous studies, which have demonstrated the influence of obesity, fractures, smoking, BMI and medications including oral corticosteroids and oral contraceptives. ${ }^{10} 38$ We were able to explore these and other risk factors with adjustment for other patient characteristics. Results highlight an interesting absence of association with VTE for traditional cardiovascular disease risk factors including hypertension and hyperlipidaemia.

Strengths of our large, long-term population-based study include the comprehensive capture of VTE risk factors and patient characteristics, allowing interrogation not only of VTE risk across multiple diseases in adjusted analysis but also assessment of independent risk factors for VTE. Exposures and outcomes were defined using algorithms previously validated in primary care. Interpretation of coefficients for individual risk factors may be limited by the potential of confounding, and these estimates do not provide a causal interpretation. ${ }^{39} \mathrm{~A}$ further limitation of the study, similar to all studies using routine data, include the potential of unmeasured confounding and selection bias. Findings may not be generalisable to more ethnically diverse populations than the UK. Despite the use of validated algorithms to classify $\mathrm{CD}, \mathrm{UC}, \mathrm{RA}$ and the use of published guidance to define PsA, the lack of medical record review and use of clinical criteria to classify these IMIDs is a further limitation of the study, since diagnoses were recorded in primary care and may not have been made by specialists. When evaluating VTE risk factors, chance findings offer a potential explanation for differences in the groups with and without an IMID due to the number of associations tested. Family history of VTE is poorly captured in primary care data, and a resultant lack of power offers the most likely explanation for the observation that family history of VTE was not a significant risk factor in the IMID cohort. Similarly, this study will have systematically under captured biologic 

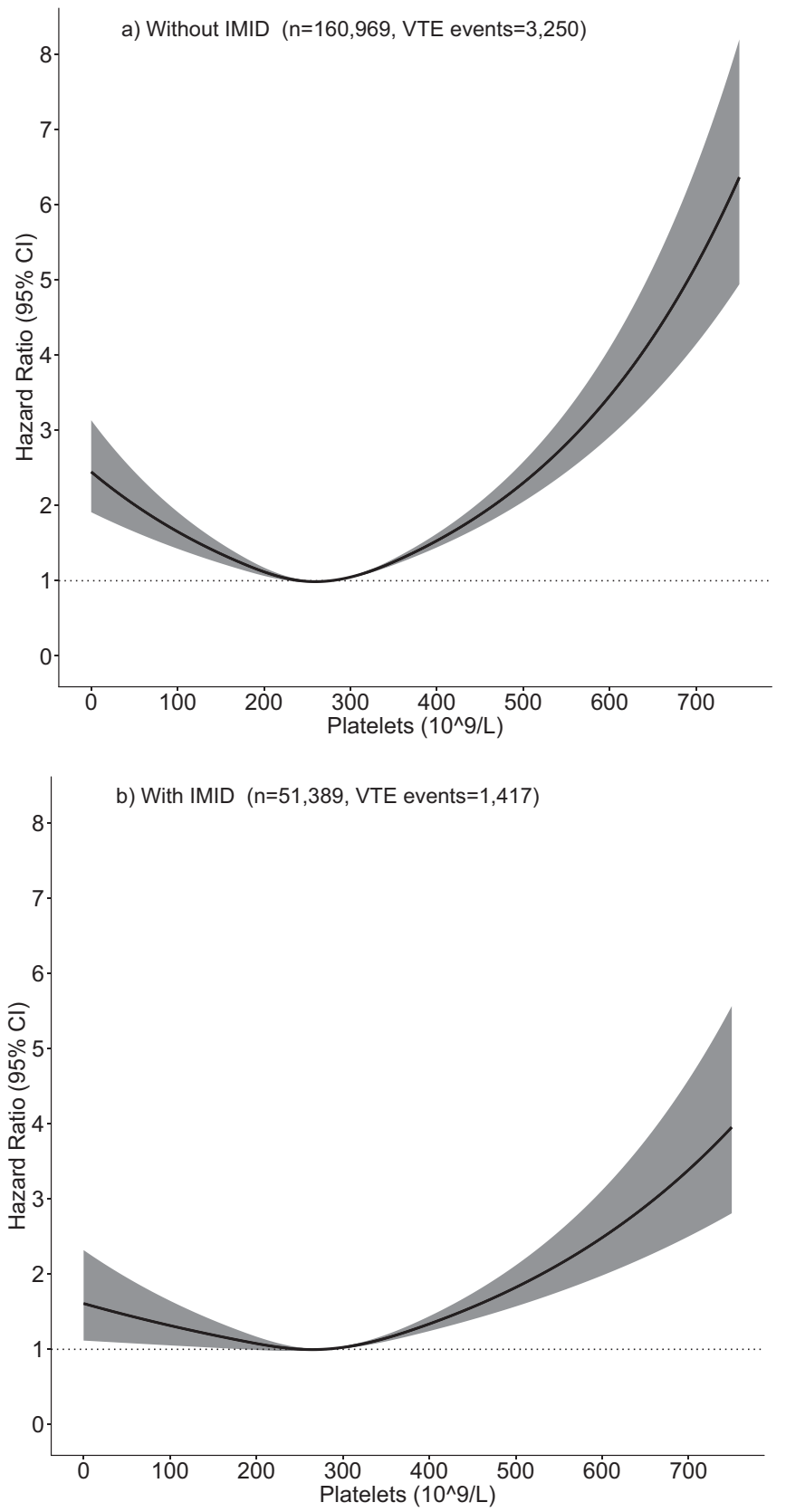

Figure 2 Association of continuous time-varying platelet count with time to venous thromboem bolism (VTE) in individuals with and without immune-mediated inflammatory diseases (IMID). Platelet count modelled using a restricted cubic spline with 3 knots in multivariable models adjusted for the same covariates as listed in Table 4, relative to the mean platelet count in individuals with an IMID $\left(277 \times 10^{9} / \mathrm{L}\right)$.

medication prescribing as, in the UK, these are prescribed by specialists and not captured in primary care. Secondary care data were not available to evaluate risk associated with surgery, an established major VTE risk factor. For analysis of time-updated platelet count, we used a complete-case approach, and for other missing information including BMI and ethnicity, we used the missing indicator variable method, as data are likely to be missing not at random meaning multiple imputation may lack validity. ${ }^{40}$

Our study is timely and of particular relevance in the context of the clinical interest in VTE in people with IMID. Our data provide an understanding of the contextual risk in IMID populations, and suggests considerable potential to update or augment existing VTE risk stratification decision aids such as the Wells Score with more refined multivariable prediction models incorporating routinely measured clinical patient characteristics. Another interesting direction for future research would be to use timeupdated risk models to evaluate the temporal association between measures of IMID disease extent and severity, and by use of medication, in particular immunosuppressive treatment, and risk of VTE. This would provide further important information for clinicians responsible for monitoring patients with IMIDs in primary care.

In summary, VTE is more common in people with UC, $\mathrm{CD}$, RA, and PsA compared with those without these diseases, highlighting the need for increased awareness among clinicians. Although associations do not have a causal interpretation, this study refines our understanding of classical VTE risk factors in people with an IMID compared with the wider population. Our data provide an initial platform for the risk assessment of individual patients with an IMID, and support active monitoring and strategies to mitigate VTE risk in people with an IMID.

\section{Author affiliations}

${ }^{1}$ Centre for Rheumatic Diseases, King's College London, London, UK

${ }^{2}$ New Road Surgery, Croxley Green, Hertfordshire, UK

${ }^{3}$ Department of Gastroenterology, Guy's and St Thomas' NHS Foundation Trust, London, UK

${ }^{4}$ School of Immunology and Microbial Sciences, King's College London, London, UK ${ }^{5}$ Pfizer Innovative Health, Tadworth, UK

${ }^{6}$ Royal College of General Practitioners Research and Surveillance Centre (RSC), London, UK

${ }^{7}$ Nuffield Department of Primary Care Health Sciences, University of Oxford, Oxford, UK

${ }^{8}$ Centre for Musculoskeletal Research, School of Biological Sciences, The University of Manchester, Manchester, UK

${ }^{9} \mathrm{NIHR}$ Manchester Biomedical Research Centre, Manchester, UK

Acknowledgements Patients and practices who are members of the Royal College of General Practitioners (RCGP) Research and Surveillance Centre (RSC) network, who allow their data to be shared for surveillance, research, quality improvement and education. The collaboration of primary care computerised medical record system providers EMIS, TPP, InPractice Systems and Apollo in facilitating the RCGP RSC data.

Contributors JG, KB, PI, KK, MN, RN, SdeL and MHB designed the study, supervised the data analysis, provided the interpretation of results, and contributed to the drafting and critical review of the manuscript. All authors approved the final draft. Medical writing and statistical support, funded by Pfizer, was provided by John Dennis (PhD), Andrew McGovern (MD) and Anita Lynam (PhD) [Momentum Data], with project management support from Filipa Ferreira (PhD) [University of Surrey]. As corresponding author, MHB attests that all listed authors meet authorship criteria and that no others meeting the criteria have been omitted. MHB is the guarantor and accepts full responsibility for the conduct of the study, had access to the data, and controlled the decision to publish.

Funding This study, and medical writing support, was funded by Pfizer. As study authors, representatives of Pfizer contributed to the design and conduct of the study, interpretation of the data; preparation, review, or approval of the manuscript; and decision to submit the manuscript for publication. 
Competing interests All authors have completed the ICMJE uniform disclosure form at URL: www.icmje.org/coi_disclosure.pdf and declare: JG has received honoraria and/or sponsorships for conferences from AbbVie, Celgene, Janssen, Pfizer and UCB. KB has received honoraria from Tillots, Thermo Fisher Scientific, Boehringer Ingelheim, Pfizer, and Yakult. PI has received lecture fees from AbbVie, Celgene, Falk Pharma, Ferring, MSD, Janssen, Pfizer, Takeda, Tillotts, Sapphire Medical, Sandoz, Shire, and Warner Chilcott; financial support for research from MSD, Pfizer, and Takeda; advisory fees from AbbVie, Arena, Genentech, Gilead, Hospira, Janssen, Lilly, MSD, Pfizer, Pharmacosmos, Prometheus, Roche, Sandoz, Samsung Bioepis, Takeda, Topivert, VH2, Vifor Pharma, and Warner Chilcott. KK, MN and RN are employees of Pfizer. SdeL is Director of the RCGP RSC, he has received funding through his universities from Eli Lilly, GSK, Astra Zeneca, MSD, Sanofi, Seqirus, and Takeda. MHB has provided expert advice and received consultant fees and/or sponsorship for conference from AbbVie, Boehringer ingelheim, Eli Lilly, EMD Serono, Gilead, Pfizer, Roche, and Sanofi and has received research grants paid to her employer from Pfizer, Roche and UCB.

Patient consent for publication This research was done without patient involvement. Patients were not invited to comment on the study design and were not consulted to develop patient relevant outcomes or interpret the results. Patients were not invited to contribute to the writing or editing of this document for readability or accuracy.

Ethics approval Study approval was granted by the Research Committee of the RCGP RSC. The study did not meet the requirements for formal ethics board review as defined using the NHS Health Research Authority research decision tool (URL: http://www.hra-decisiontools.org.uk/research/).

Provenance and peer review Not commissioned; externally peer reviewed.

Data availability statement № additional data are available from the authors although Royal College of General Practitioners (RCGP) Research and Surveillance Centre (RSC) United Kingdom primary care data are available by application to the Research Committee of the RCGP RS.

Supplemental material This content has been supplied by the author(s). It has not been vetted by BMJ Publishing Group Limited (BMJ) and may not have been peer-reviewed. Any opinions or recommendations discussed are solely those of the author(s) and are not endorsed by BMJ. BMJ disclaims all liability and responsibility arising from any reliance placed on the content. Where the content includes any translated material, BMJ does not warrant the accuracy and reliability of the translations (including but not limited to local regulations, clinical guidelines, terminology, drug names and drug dosages), and is not responsible for any error and/or omissions arising from translation and adaptation or otherwise.

Open access This is an open access article distributed in accordance with the Creative Commons Attribution Non Commercial (CC BY-NC 4.0) license, which permits others to distribute, remix, adapt, build upon this work non-commercially, and license their derivative works on different terms, provided the original work is properly cited, appropriate credit is given, any changes made indicated, and the use is non-commercial. See: http://creativecommons.org/licenses/by-nc/4.0/.

ORCID iDs

Maya H Buch http://orcid.org/0000-0002-8962-5642

\section{REFERENCES}

1 Walker AJ, Card TR, West J, et al. Incidence of venous thromboembolism in patients with cancer - a cohort study using linked United Kingdom databases. Eur J Cancer (Oxford, England: 1990) 2013;49:1404-13.

2 Cohen AT, Agnelli G, Anderson FA, et al. Venous thromboembolism (VTE) in Europe. The number of VTE events and associated morbidity and mortality. Thromb Haemost 2007;98:756-64.

3 Winter MP, Schernthaner GH, Lang IM. Chronic complications of venous thromboembolism. J Thromb Haemost 2017:15:1531-40.

4 Branchford BR, Carpenter SL. The role of inflammation in venous thromboembolism. Frontiers Pediatrics 2018;6:142-42.

5 Choi HK, Rho Y-H, Zhu Y, et al. The risk of pulmonary embolism and deep vein thrombosis in rheumatoid arthritis: a UK population-based outpatient cohort study. Ann Rheum Dis 2013;72:1182-7.

6 Ogdie A, Kay McGill N, Shin DB, et al. Risk of venous thromboembolism in patients with psoriatic arthritis, psoriasis and rheumatoid arthritis: a general population-based cohort study. Eur Heart J 2018;39:3608-14.
7 Grainge MJ, West J, Card TR. Venous thromboembolism during active disease and remission in inflammatory bowel disease: a cohort study. Lancet 2010;375:657-63.

8 Murthy SK, Nguyen GC. Venous thromboembolism in inflammatory bowel disease: an epidemiological review. Am J Gastroenterol 2011;106:713.

9 Mansour R, Azrielant S, Watad A, et al. Venous thromboembolism events among RA patients. Mediterr J Rheumatol 2019;30:38-43.

10 Huerta C, Johansson S, Wallander M, et al. Risk factors and short-term mortality of venous thromboembolism diagnosed in the primary care setting in the United Kingdom. Arch Intern Med 2007;167:935-43.

11 Dregan A, Charlton J, Chowienczyk P, et al. Chronic inflammatory disorders and risk of type 2 diabetes mellitus, coronary heart disease, and stroke. Circulation 2014;130:837-44.

12 Zakai NA, Wright J, Cushman M. Risk factors for venous thrombosis in medical inpatients: validation of a thrombosis risk score. J Thromb Haemost 2004;2:2156-61.

13 Harries AD, Fitzsimons E, Fifield R, et al. Platelet count: a simple measure of activity in Crohn's disease. Br Med J (Clin Res Ed) 1983;286:1476

14 Farr M, Scott DL, Constable TJ, et al. Thrombocytosis of active rheumatoid disease. Ann Rheum Dis 1983;42:545-9.

15 Scoville EA, Konijeti GG, Nguyen DD, et al. Venous thromboembolism in patients with inflammatory bowel diseases: a case-control study of risk factors. Inflamm Bowel Dis 2014;20:631-6.

16 Correa A, Hinton W, McGovern A, et al. Royal college of general practitioners research and surveillance centre (RCGP RSC) sentinel network: a cohort profile. BMJ Open 2016;6:4.

17 Kumar S, de Lusignan S, McGovern A, et al. Ischaemic stroke, haemorrhage, and mortality in older patients with chronic kidney disease newly started on anticoagulation for atrial fibrillation: a population based study from UK primary care. BMJ 2018;360.

18 Williams R, Alexander G, Armstrong I, et al. Disease burden and costs from excess alcohol consumption, obesity, and viral hepatitis: fourth report of the lancet standing commission on liver disease in the UK. Lancet (London, England) 2018;391:1097-107.

19 Woodmansey C, McGovern AP, McCullough KA, et al. Incidence, demographics, and clinical characteristics of diabetes of the exocrine pancreas (type 3c): a retrospective cohort study Diabetes Care. 2017:40: 1486-93.

20 Nikiphorou E, de Lusignan S, Mallen C, et al. Haematological abnormalities in new-onset rheumatoid arthritis and risk of common infections: a population-based study. Rheumatology 2019.

21 Stapley SA, Rubin GP, Alsina D, et al. Clinical features of bowel disease in patients aged $<50$ years in primary care: A large case-control study. Br J Gen Pract 2017;67:e336-e44.

22 Abrahami D, Douros A, Yin H, et al. Dipeptidyl peptidase-4 inhibitors and incidence of inflammatory bowel disease among patients with type 2 diabetes: population based cohort study. BMJ (Clin Res Ed) 2018;360.

23 Muller S, Hider SL, Raza K, et al. An algorithm to identify rheumatoid arthritis in primary care: a clinical practice research datalink study. BMJ Open 2015;5:e009309.

24 Thomas SL, Edwards CJ, Smeeth L, et al. How accurate are diagnoses for rheumatoid arthritis and juvenile idiopathic arthritis in the general practice research database? Arthritis Rheum 2008:59:1314-21.

25 Lewis JD, Brensinger C, Bilker WB, et al. Validity and completeness of the general practice research database for studies of inflammatory bowel disease. Pharmacoepidemiol Drug Saf 2002;11:211-8.

26 Dave S, Petersen I. Creating medical and drug code lists to identify cases in primary care databases. Pharmacoepidemiol Drug Saf 2009;18:704-7.

27 de Lusignan S, Liaw ST, Michalakidis G, et al. Defining datasets and creating data dictionaries for quality improvement and research in chronic disease using routinely collected data: an ontology-driven approach. Inform Prim Care 2011;19:127-34.

28 Lawrenson R, Todd JC, Leydon GM, et al. Validation of the diagnosis of venous thromboembolism in general practice database studies. $\mathrm{Br}$ J Clin Pharmacol 2000;49:591-6.

29 Department for Communities and Local Government. The English indices of deprivation. [Internet]. 2015. Available https://www.gov.uk/ government/statistics/english-indices-of-deprivation-2015 (accessed 16 Jun 2019)

30 Tippu Z, Correa A, Liyanage $\mathrm{H}$, et al. Ethnicity recording in primary care computerised medical record systems: an ontological approach. $J$ Innovation Health Inf 2017;23:920.

31 McGovern A, Hinton W, Correa A, et al. Real-world evidence studies into treatment adherence, thresholds for intervention and disparities in 
treatment in people with type 2 diabetes in the UK. BMJ Open 2016;6:11.

32 Hinton W, McGovern A, Coyle R, et al. Incidence and prevalence of cardiovascular disease in English primary care: a cross-sectional and follow-up study of the royal college of general practitioners (RCGP) research and surveillance centre (RSC). BMJ Open 2018;8:8.

33 Mantha S, Karp R, Raghavan V, et al. Assessing the risk of venous thromboembolic events in women taking progestin-only contraception: a meta-analysis. BMJ 2012;345:e4944-e44.

34 Sutcliffe D, Lester H, Hutton J, et al. NICE and the quality and outcomes framework (QOF) 2009-2011. Qual Prim Care 2012;20:47-55.

35 Ungprasert P, Srivali N, Spanuchart I, et al. Risk of venous thromboembolism in patients with rheumatoid arthritis: a systematic review and meta-analysis. Clin Rheumatol 2014;33:297-304.
36 Bester J, Pretorius E. Effects of IL- 1 beta, IL- 6 and IL-8 on erythrocytes, platelets and clot viscoelasticity. Sci Rep 2016;6:32188.

37 Msaouel P, Lam AP, Gundabolu K, et al. Abnormal platelet count is an independent predictor of mortality in the elderly and is influenced by ethnicity. Haematologica 2014;99:930-6.

38 Cheng YJ, Liu ZH, Yao FJ, et al. Current and former smoking and risk for venous thromboembolism: a systematic review and meta-analysis. PLoS Med 2013;10:e1001515.

39 Westreich D, Greenland S. The table 2 fallacy: presenting and interpreting confounder and modifier coefficients. Am J Epidemiol 2013;177:292-8.

40 Marston L, Carpenter JR, Walters KR, et al. Issues in multiple imputation of missing data for large general practice clinical databases. Pharmacoepidemiol Drug Saf 2010;19:618-26. 